Published in final edited form as:

Exp Gerontol. 2012 October ; 47(10): 803-806. doi:10.1016/j.exger.2012.06.004.

\title{
The Evolution of Transfers and Life Histories
}

\author{
Ronald Lee and \\ Departments of Demography and Economics, University of California, 2232 Piedmont Ave, \\ Berkeley, CA 94720, 1-510-540-8986 (phone), 1-151-540-5367 (fax) \\ C. Y. Cyrus \\ Chu Institute of Economics, Academia Sinica, 128 Academia Road Sec. 2, Nankang, Taipei, \\ TAIWAN \\ Ronald Lee: rlee@demog.berkeley.edu; C. Y. Cyrus: cyruschu@gate.sinica.edu.tw
}

\begin{abstract}
Much of life history theory analyzes life histories of independent, isolated individuals, who grow, forage, reproduce, and die. However, in many species social interactions such as food sharing are a key part of the life history strategy, altering the energetic budget constraint. Transfers and sharing raise reproductive success and also alter the fitness impact of other aspects of the life history. We discuss a variety of traits and behaviors for which transfers are important, synthesizing results from a number of earlier papers. Topics include the U-shaped mortality curve, post reproductive survival, causes of early life mortality decline, why intergenerational transfers evolve and co-evolve with longevity, time preference, sexual dimorphism and sexual differences in transfers, menopause, demographic advantages of social sharing, and consequences of social sharing for life history evolution.
\end{abstract}

\section{Keywords}

transfers; intergenerational; menopause; mortality; sexual dimorphism; life history

\section{Introduction}

Much of life history theory analyzes independent, isolated individuals who grow, forage, reproduce, and die. However, in many species individuals interact in ways that increase reproductive success, such as parental care of offspring or broader forms of social cooperation or sharing. These behaviors then alter the fitness impact of other aspects of the life history and expand the life history options by altering the energetic budget constraint. Transfers of energy or time are a key aspect of these behaviors. Some transfers are intergenerational, between adults and their descendants. Other transfers can occur among related or unrelated individuals of the same generation. In humans, as in many species, these vertical and horizontal aspects of sociality are intertwined. Humans invest heavily in a small number of offspring who are nutritionally dependent on adults until around age 20 (Kaplan, 1994). Provisioning is mainly by parents, but there are periods in the family life cycle when the child dependency burden exceeds parental capacities and broader social transfers are

(C) 2012 Elsevier Inc. All rights reserved.

Correspondence to: Ronald Lee, rlee@demog . berkeley . edu.

Publisher's Disclaimer: This is a PDF file of an unedited manuscript that has been accepted for publication. As a service to our customers we are providing this early version of the manuscript. The manuscript will undergo copyediting, typesetting, and review of the resulting proof before it is published in its final citable form. Please note that during the production process errors may be discovered which could affect the content, and all legal disclaimers that apply to the journal pertain. 
necessary. Hill and Hurtado (2009) summarize: "food provisioning is ubiquitous, generally biased in favour of helping families with large dependency loads and not limited to kin assistance".

Here we synthesize our papers that analyze the conditions under which intergenerational transfers or social sharing may evolve, and we consider how these transfers and sharing influence the coevolution of other life history traits including fertility, mortality, time preference, the intergenerational division of labor, menopause, and sexual dimorphism. Our analytic approaches include fitness impacts, microsimulation, and optimal life histories. We emphasize our own theoretical work, and regrettably do not have space for a balanced overview of all research on these topics.

We explicitly model intergenerational transfers of food, subject to a social budget constraint based on the average population age distribution in the cooperative breeding group rather than on the presence of particular biological relatives. This approach is consistent with some empirical and ethnographic studies of humans that find that food is shared within small groups, often with little or no kin-bias, and that relatives and friends other than parents and grandparents often make transfers or share in child care (Hrdy, 2009; Sear and Mace, 2008; Hill and Hurtado, 2009). The special importance of the mother is not reflected in this approach, and our approach is less consistent with other empirical research that finds a strong kin-bias in intergenerational transfers (Gurven, 2004; Gurven et al. 2000; Gurven et al, 2002). Kin-bias would strengthen our substantive conclusions but undermine our analytic use of stable populations.

\section{The Basic Optimal Life History Model}

In our optimal analysis (Chu et al, 2010), we view natural selection as tending to maximize reproductive fitness as measured by the intrinsic rate of natural increase or sometimes the Net Reproduction Rate, subject to an energy constraint. The constraint $f$ describes the total energy required to achieve a given combination of life history traits at a particular age: mortality risk, fertility risk, and body growth. $f$ is generally nonlinear, reflecting possible diminishing returns to investment in reducing mortality $\left(\mu_{a}\right)$, raising fertility $\left(m_{a}\right)$, or accelerating body growth $\left(z_{a}\right)$, where $w_{a}$ is the body size attained by age $a$ (or other form of somatic capital, see Kaplan and Robson, 2002, and Robson and Kaplan, 2003). A foraging function $\zeta_{a}$ describes the available energy (net of energy costs of foraging) depending on the body size (or other somatic measure such as brain size) at each age $a$. The energy constraint defines the life history trait tradeoff frontier at each age.

$$
f_{a}\left(\mu_{a}, m_{a}, z_{a}\right) \leq \zeta_{a}\left(w_{a}\right), \quad \forall a
$$

The partial derivative of the tradeoff function at age $x$ with respect to fertility $m, f_{X, m}$ (for example), is the energy cost of raising fertility at age $x$. If $f$ were linear, this derivative would just be the cost coefficient on fertility, giving the constant cost of raising fertility by one birth.

The literature has established that if $f$ is linear (as in Chu and Lee, 2006), the optimal life history exhibits determinate growth: until some age $J$ the organism invests in growth and maintenance/survival but not in fertility; after $J$ it invests in maintenance/survival and fertility but not in growth. With a nonlinear tradeoff function the optimal life history is not necessarily determinate growth. However, most organisms that make substantial intergenerational transfers, such as mammals and birds, do have determinate growth. Even with determinate growth, foraging output might continue to increase for a while after age $J$ due to accumulating experience and knowledge (Promislow, 1991). 
Consider a determinate growth organism in which an adult at age $a$ transfers a total amount $T_{a}$ to young, while a young individual at age a receives a total transfer (from adults of all ages) of $R_{a}$. Now the new budget constraints are:

$$
\begin{gathered}
f_{a}\left(\mu_{a}, m_{a}, 0\right) \leq \zeta_{a}\left(w_{r}\right)-T_{a}, \quad \text { for adults } \\
f_{a}\left(\mu_{a}, 0, z_{a}\right) \leq \zeta_{a}\left(w_{a}\right)+R_{a}, \quad \text { for juveniles }
\end{gathered}
$$

These constraints will depend on environmental context, presence of predator and prey species, competitors, and so on. The social budget constraint requires that the populationweighted sum of $T_{a}$ equals the population weighted sum of $R_{a}$.

\section{Optimal Juvenile Mortality}

The first order condition for an optimal juvenile mortality trajectory is that the marginal energy cost of reducing mortality at age a must equal the net marginal fitness gain of reducing mortality at that age:

$$
-f_{a, \mu}=\frac{\int_{J}^{\omega} e^{-\rho x} l_{x} m_{x} d x+\int_{J}^{\omega} \frac{1}{f_{x, m}} \int_{0}^{a} e^{-\rho s} l_{s} R_{s, x} d s d x}{\frac{1}{f_{a, z}} \exp \left(\int_{a}^{J} \frac{\zeta_{x, w}}{f_{x, z}} d x\right) \int_{r}^{\infty} e^{-\rho x} l_{x} \frac{\zeta_{x, w}}{f_{x, m}} d x}=\frac{M+\tilde{T}_{a}}{K_{a} N}
$$

Here $\rho$ is the intrinsic rate of natural increase, and letters at the far right name the corresponding integrals. This complicated expression is not an explicit solution for the age path of juvenile mortality, because $I_{X}$ (survival from birth to age $\mathrm{x}$ ) also occurs on the right side of the equation in the various terms. However, this expression helps us understand the shape of the optimal age trajectory of juvenile mortality and the forces generating it.

The LHS is the cost of reducing the death rate at age $a$, that is the negative of the "cost" (or energy released) of raising mortality. On the RHS, M is the expected lifetime births for an individual who survives to the age of reproductive maturity, $J . M$ does not vary by age before $J$. Because $M$ is the sole influence on juvenile mortality in Hamilton's (1966) theory, it predicts that juvenile mortality is low and constant across juvenile ages, in contrast to Fisher's (1930) result that juvenile mortality falls until the age of reproductive maturity based on reproductive value. $\tilde{T}_{a}$ is the expected total energy to be transferred by an age $a$ individual over the remainder of life, expressed in units of the fertility cost of those transfers, as is $M$. This equals the fertility value of all the transfers $R$ received by the birth cohort up to age $a$, per juvenile surviving to age $a$, including the wasted transfers to other juveniles who died before reaching age $a$.

The RHS denominator includes $K_{a}$, which is the effect of an additional unit of energy invested in body weight at age $a$ on mature size at age $J$. $N$ is the effect of an increase in body weight at age $J$ on remaining lifetime fertility, an effect which is independent of juvenile age. So the product $K_{a} N$ is the lifetime fertility cost of spending a unit of energy on reducing mortality rather than on raising body weight.

As a rises from 0 to $J, M$ remains constant, $T_{a}$ rises, and $K_{a} N$ declines. The right hand side, therefore, rises with age. Thus the energetic cost of reducing mortality rises with juvenile age in the optimal life history, and from this we can infer that the level of mortality declines with juvenile age, from 0 to $J$. This decline reflects the Hamilton Effect, Transfer Effect, 
Compounded Effect of Growth, and any variations by age in the tradeoff cost of reducing mortality, $-f_{a, \mu}$.

\section{Optimal Adult Mortality}

For adult mortality, the corresponding first order condition for an optimum (see Chu et al, 2008: equation 9) is:

$$
-f_{a, \mu}=\frac{e^{\rho a} f_{a, m}}{l_{a}}\left[\int_{a}^{\infty} e^{-\rho x} l_{x} m_{x} d x+\int_{a}^{\infty} e^{-\rho x} l_{x} \frac{T_{x}}{f_{x, m}} d x\right]=\frac{e^{\rho a} f_{a, m}}{l_{a}}\left(M_{a}+\tilde{T}_{a}\right)
$$

Here $M_{a}+\tilde{T}$ represents the gross (ignoring costs) benefit of investing in continuing adult survival. $M_{a}$ is expected future fertility (the Hamilton effect) and $\tilde{T}_{a}$ is the expected cumulated transfers to be made above age $a$ as in Lee (2003), expressed in units of fertility to convert to units commensurate with $M_{a}$. The $M_{a}+\tilde{T}$ in the numerator corresponds closely to the weighted average of the Hamilton and transfer effects as in Lee (2003). As noted by Hamilton, $M_{a}$ goes to zero as a approaches menopause.

However, the transfer term $\widetilde{T}_{a}$ continues positive past the reproductive ages, reflecting the transfers that older men and women continue to make in hunter-gatherer societies. This component indicates that human postreproductive survival is explained by the continuing role of postreproductive adults in assisting the reproductive efforts of their offspring and other kin through transfers of energy.

However, there are important differences from Lee (2003). First, $M_{a}+\tilde{T}$ is multiplied by the energy cost of fertility at age $a$, which we expect to rise with age due to deterioration of oocytes and general aging. Second, the RHS is calculated conditional on surviving to age a $\left(I_{X} / I_{a}\right)$ in the optimal approach, whereas in Hamilton and in Lee, multiplication is by $I_{X}$ alone. This reflects a key difference between the mutation accumulation-strength of selection approach and the optimization approach based on positive selection. The optimization approach is always forward looking, so that the optimal approach is chosen for age $a$ conditional on having reached age $a$. But in the mutation accumulation-deselection approach, effects are evaluated at birth, and consequences that are farther in the future at more advanced ages are consequently discounted.

Only by an extended argument can it be concluded from (1.5) that adult mortality will generally increase with age. There can be a flat or declining portion of the adult mortality schedule following reproductive maturity, if accumulating experience leads to increased foraging efficiency or care of offspring with age or to more efficient evasion of predators (Promislow, 1991; Vaupel et al 2004).

\section{The Evolution of Transfers}

An evolved pattern of intergenerational transfers functions something like a credit market, enabling an organism to "borrow" resources when young to grow faster and mature earlier or to invest in a costly brain, at the cost of repaying the debt in adulthood through corresponding transfers to its own offspring or other young. As with any transfer system, in steady state the rate of return equals the rate of population growth which in equilibrium will be 0 . Transfers received when young, for example, must exactly equal the survival-weighted expected value of transfers made when old. Some organisms like humans make substantial transfers of this sort, necessarily having fewer of these high-cost offspring, that is, low fertility. Other organisms have less or no parental care, limiting their investment to little beyond the gametes themselves, like an orchid that may produce a billion seeds. 
Consider the parental transfer of a unit of energy that is shared among all offspring. Would it be fitness enhancing? That depends on how much the lifetime fitness of the each offspring is raised and how much the fitness of the parent is reduced. The offspring's fitness gain depends on the efficiency with which the energy can be used to increase growth and on how much the resulting increase in size would raise the lifetime reproductive success of the offspring (see Chu et al, 2010:12; Kaplan and Robson, 2002; and Robson and Kaplan, 2003). The parent's fitness cost depends directly on the size of the total transfer and inversely on the parent's marginal cost of fertility, which generally rises with age in species with determinate growth. The parental transfer will be diluted by the number of offspring at the age to which the parent is making transfers, which depends in part on the survival probability from that offspring age to the parent's age. If new transfers would enhance fitness, then we expect them to continue to evolve until, in the limit, there is zero advantage for lifetime fitness in further transfers (Chu and Lee, 2006; Chu et al, 2010). Longevity and transfers should co-evolve (Chu and Lee, 2006; Chu et al, 2010; Carey and Judge, 2001).

\section{Time Preference}

Organisms frequently must choose whether to undertake costly actions with a payoff that may be relatively far in the future. Such choices are governed by "time preference", which is arguably an evolved trait. Suppose we take away X units of energy from an individual at age $a$ and compensate her with $\mathrm{Y}$ units of energy $t$ years later at age $a+t$ (if she survives) such that her lifetime fitness is unchanged-that is, we move along an isofitness contour. $\mathrm{Y} / \mathrm{X}$ is the marginal rate of substitution (MRS) between age $a$ and age $a+t$, and $\ln (\mathrm{Y} / \mathrm{X}) / \mathrm{t}$ is the rate of time preference between age $a$ and $a+t$. We would expect an organism to "take out a loan" (that is, evolve intergenerational transfers) if the interest rate is less than the rate of time preference, and conversely (Rogers, 1994; Sozou and Seymour, 2003; Robson and Samuelson, 2009). Chu et al (2010) establish new results by analyzing the case of determinate growth with intergenerational transfers. Like the previous literature, the rate of time preference between adult ages equals the force of mortality plus the rate of increase of the cost of fertility plus the population growth rate. This rate of time preference will eventually rise following maturity in line with mortality and the costs of fertility. But within the juvenile ages mortality does not matter, nor does the cost of fertility nor the population growth rate, and instead time preference is determined by differences in the ability to convert energy into growth (size, brain), and to convert body size into energy (see denominator of (1.4)). The net effect is that time preference follows a $\mathrm{U}$ pattern over the lifecycle, starting high, declining to around maturity, and then rising in adulthood.

If intergenerational transfers evolve, we might expect that eventually they would result in the MRS between all pairs of ages being unity and the rate of time preference being zero, in line with the rate of return to the transfer system. If the MRS were greater than one, then evolving transfers could increase fitness by moving energy between the two ages. However, we argue that transfers would not evolve to this point, due to paternity uncertainty and the constant risk that parental mortality would cause offspring death, when transfers are high and lengthy.

\section{Evolution of Food Sharing}

For humans, prolonged and intensive investment in juvenile development confers benefits, but also poses demographic risks and inefficiencies. 1) Parents risk losing all the cumulated investments in any children who are still dependent at the time of parental death. One potential solution is life insurance through social sharing, such that if a mother dies, other kin or non-kin would raise the offspring. 2) During the family life cycle there are periods when parental foraging can provide more than enough food, but other periods when the 
number of dependent children is too high and the parents might be unable to provision the entire family on their own (Hill and Hurtado, 2009; Kaplan and Gurven, 2006). This systematic variation in the dependency ratio over the family life cycle could be smoothed if multiple families lived together and shared food. 3) Because birth and survival are partially random, some families have light dependency burdens and others heavy ones. Once again, averaging across these random states through social sharing among multiple families offers increased efficiency and higher average fitness. These issues are explored in Lee (2008). When food sharing groups include non-nuclear kin, distant kin and non-kin, then the fitness impacts of various other life history traits is altered, redirecting the evolution of life history traits. If the group bears part of the cost of provisioning and perhaps caring for offspring, then the fitness penalty for individually high fertility will be reduced, and higher fertility will tend to evolve — a kind of evolutionary reproductive free riding.

Furthermore, if a child dies, the expected future group contributions for that child are lost to the parent's family. However, in the case with no cross-family sharing, the dead child's family recovers all the future expenditures that the dead child no longer needs. Therefore, a child death is more costly under social sharing, so lower infant and child mortality will evolve in addition to higher fertility. These altered levels of fertility and mortality are inefficient compared to the individually optimal levels or the levels under a coordinated group choice. Similarly, the selective pressure for postreproductive survival is weakened, since there are substitutes for parental care. Therefore evolved adult and postreproductive survival will be inefficiently lower than in the absence of sharing. Finally, social sharing creates incentives to renege on implicit social contracts, for example by killing a child if its parent dies (Hill and Hurtado, 1996). These issues are explored in Lee (2008).

\section{Evolution of Menopause}

Juveniles require transfers of time for guarding, training, and general care as well as transfers of food. Care time is provided mainly by younger and older adults, although older siblings may also help (Lee and Kramer, 2002). Younger and older adults make transfers of both care and food to their offspring, but with different efficiency. Experience and knowledge increase with age, but physical strength declines. Empirically, either younger or older women will have a comparative advantage (greater relative efficiency) in foraging over caring. So long as comparative efficiencies differ, there is room for fitness gains through a division of labor between younger and older women. The outcome will also be influenced by age differences in the physiological efficiency of reproduction $\left(f_{X, m}\right)$, for example rising fertility costs due to deterioration of the oocytes. The rising risk of orphanhood for late born offspring may similarly be a factor.

In the evolving division of labor, younger women may move toward higher fertility and shorter birth intervals and also to more foraging time, while older women move toward lower fertility and assisting their daughters with more care time, while still foraging for their own and some of their children's consumption. Eventually this evolving division of labor may reduce the grandmother's fertility to zero: menopause. However, if the older woman still has a comparative advantage in giving care, the division of labor might continue to evolve, with the grandmother's foraging time reduced to her own subsistence needs. Further specialization could occur only with food transfers to the grandmother, enabling her to reduce her foraging time to concentrate on additional childcare. We show that this situation can arise only after menopause has evolved (Chu and Lee, 2010). In the real world, younger old women may in fact specialize in foraging rather than care, as in the Grandmother Hypothesis. However, older old women do switch to specialization in child care. This sort of specialization in foraging or caring is seen in some cooperatively breeding mammals (e.g. African hunting dogs, naked mole rats) where only the dominant female breeds while other 
females hunt and provision her and the offspring, but this behavior is not strictly age-based and the nonbreeding females only temporarily lose their reproductive capacity. There is also a loose similarity to eusocial insect species where there is a division of labor between reproduction and fertility, and perhaps between foraging and care giving.

\section{Sexual Dimorphism}

Following Darwin (1871), sexual dimorphism and sexual selection arise because the male cost of producing sperm is trivially small compared to the female cost of producing eggs, a difference that is magnified by the cost of pregnancy and delivery for viviparous species, and for birds. This means that males have a much higher Potential Rate of Reproduction (PRR) than females, so that actual male reproduction is constrained by the female costs. At the female determined rate, the males have substantial surplus energy. Chu and Lee (2011) formalize this theory and derive additional implications. One possible evolutionary outcome is monogamous pair bonding, with relatively certain paternity and substantial paternal transfers to offspring. We focus mainly on a different possibility, that surplus energy of males is used in male-male intrasexual competition for reproductive access to females, such that some males do not mate at all and others mate with multiple females. In this case, the surplus energy of males may be devoted to weaponry and body size for physical combat, or to larger testicles for sperm competition, or to elaborate but largely symbolic body parts, or to costly display behaviors: that is, to sexual dimorphism. The direction of these evolved investments for male intrasexual mate competition is largely determined by female preferences, which determine the male characteristics that raise the probability of access to females and lead to sexual selection.

In this scenario, successful males father on average $\mathrm{N}$ offspring which is $\mathrm{k}$ times as many as the average female bears. In equilibrium, males have equal chances and winners are determined by a "lottery." For the unsuccessful males, the incentive to transfer to nonoffspring is likely smaller than the incentive to invest in competition. For a father, transferring q units per offspring to $\mathrm{N}$ offspring would cost $\mathrm{Nq}$, which is $\mathrm{k}$ times as costly as a similar transfer per offspring by a female. This greater number of offspring for fathers dilutes the incentive for male transfers per child. After dilution, this incentive to transfer may be smaller than the opportunity cost, that is, the foregone investment in intrasexual competition and in protecting offspring from the unsuccessful males. For these reasons, males make smaller transfers than females. For related reasons, males never experience menopause: very little male energy would be saved by terminating sperm production, and males make little or no transfers, so selection for postreproductive survival would be slight. However, the case of male humans is different: they do experience a social cessation of childbearing around the age their female mates do while they continue to produce extensive net surplus to provision their grand-offspring and adult offspring (Kaplan et al, 2010).

\section{Conclusions}

Intergenerational transfers expand life history options by shifting energy from older ages where it has lower marginal fitness value to younger ages where fitness value is higher through investment in growth. Consequently rates of time preference are reduced for a transferring organism. Extensive transfers lead to demographic risks and inefficiencies which can be reduced through social sharing, but this alters the evolution of life history traits. Transfer patterns may also coevolve with age patterns of adult fertility, leading to menopause. Finally, the low direct cost of male fertility means population growth is limited by female costs, leaving males with surplus that can be invested in sexually dimorphic traits that appeal to females. If successful males mate with multiple females, the incentives for 
male transfers to offspring are reduced, perhaps to zero with available male energy devoted to further intrasexual competition.

\section{Acknowledgments}

Lee's research for this paper was funded by NIA grants P01 AG022500 and R37-AG025247.

\section{References}

1. Carey JR, Judge DS. Life Span Extension in Humans is Self-reinforcing: A General Theory of Longevity. Popul Dev Rev. 2001; 27:3,411-30.

2. Chu CYC, Lee RD. The Coevolution of Intergenerational Transfers and Longevity: An Optimal Life History Approach. Theor Popul Biol. 2006; 69:193-201. [PubMed: 16406044]

3. Chu CYC, Chien H-k, Lee R. Explaining the Optimality of U-shaped Age-specific Mortality. Theor Popul Biol. 2008; 73:171-180. [PubMed: 18178233]

4. Chu, CY.; Lee, RD. On the Evolution of Filial Piety. 2010. submitted

5. Chu CYC, Chien H-k, Lee R. The Evolutionary Theory of Time Preferences and Intergenerational Transfers. J Econ Behav Organ. 2010; 76:3,451-464.10.1016/j.jebo.2010.09.011

6. Chu, CYC.; Lee, RD. Sexual Dimorphism and Sexual Selection: A Unified Economic Analysis. 2011. submitted

7. Darwin, C. The Descent of Man, and Selection in Relation to Sex. London: John Murray; 1871.

8. Fisher, RA. The Genetic Theory of Natural Selection. Clarendon Press; Oxford: 1930.

9. Gurven M. To give and to give not: The behavioral ecology of human food transfers. Behavioral and Brain Sciences. 2004; 27(4):543-583.

10. Gurven M, Hill K, Kaplan H. From forest to reservation: transitions in food sharing behavior among the Ache of Paraguay. Journal of Anthropological Research. 2002; 58(1):93-120.

11. Gurven M, Hill K, Kaplan H, Hurtado M, Lyles R. Food transfers among Hiwi foragers of Venezuela: Tests of reciprocity. Human Ecology. 2000

12. Hamilton WE. The Molding of Senescence by Natural Selection. Theor Popul Biol. 1966; 12:1245.

13. Hill K, Hurtado A. Cooperative breeding in South American hunter-gatherers. Proc of the Royal Society. 2009; 276(1674):3863-3870.10.1098/rspb.2009

14. Hill, K.; Hurtado, MA. Ache Life History. Aldine de Gruyter; New York: 1996.

15. Hrdy, S. Mothers and Others: The Evolutionary Origins of Mutual Understanding. Harvard University Press; Cambridge: 2009.

16. Kaplan H. Evolutionary and Wealth Flows Theories of Fertility: Empirical Tests and New Models. Popul Dev Rev. 1994; 20:4,753-791.

17. Kaplan H, Gurven M, Winking J, Hooper P, Stieglitz J. Learning, menopause and the human adaptive complex. Annals of New York Academy of Sciences. 2010; 1204:30-42.

18. Kaplan HS, Gurven M. Determinants of Time Allocation Across the Lifespan: A Theoretical Model and an Application to the Machiguenga and Piro of Peru. Human Nature. 2006; 17:1,1-49.

19. Kaplan HS, Robson AJ. The emergence of humans: the coevolution of intelligence and longevity with intergenerational transfers. Proc Natl Acad Sci U S A. 2002:10221-10226. [PubMed: 12122210]

20. Lee RD. Rethinking the evolutionary theory of aging: transfers, not birth, shape senescence in social species. Proc Natl Acad Sci U S A. 2003; 100:9637-9642. [PubMed: 12878733]

21. Lee R. Sociality, Selection and Survival: simulated evolution of mortality with intergenerational transfers and food sharing. Proc Natl Acad Sci U S A. 2008 published May 5, 2008. (Social Sciences). 10.1073/pnas.0710234105

22. Lee R, Kramer K. Children's Economic Roles in the Context of the Maya Family Life Cycle: Cain, Caldwell, and Chayanov Revisited. Popul Dev Rev. 2002; 28:3,475-499.

23. Promislow D. Senescence in Natural Populations of Mammals: A Comparative Study. Evolution. 1991; 45:1869-1887. 
24. Robson AJ, Kaplan HS. The Evolution of Human Longevity and Intelligence in Hunter-Gatherer Economies. Am Econ Rev. 2003; 93:150-169.

25. Robson A, Samuelson L. The Evolution of Time Preference with Aggregate Uncertainty. Am Econ Rev. 2009; 99:1925-53.

26. Rogers AR. Evolution of Time Preferences by Natural Selection. Am Econ Rev. 1994; 84:460481.

27. Sear R, Mace R. Who keeps children alive? A review of the effects of kin on child survival. Evolution \& Human Behavior. 2008; 29:1,1-18.

28. Sozou PD, Seymour R. Augmented Discounting: Interaction between Ageing and Time-preference Behavior. Proc Biol Sci. 2003; 270:1047-1053. [PubMed: 12803894]

29. Vaupel JW, Baudisch A, Dolling M, Roach DA, Gampe J. The Case for Negative Senescence. Theor Popul Biol. 2004; 65:339-351. [PubMed: 15136009] 


\section{Highlights}

- Intergenerational transfers alter the lifetime energetic budget constraint.

- Transfers co-evolve with and shape other life history traits.

- Transfers help explain postreproductive survival, dimorphism, time preference.

- Social sharing also shapes evolution of fertility and mortality by age.

- Transfers and sharing can raise efficiency and also cause inefficient distortions. 